Nadine Cerf-Bensussan Bana Jabri

\section{ADRESSES}

N. Cerf-Bensussan : Inserm-EPI9925, Faculté Necker, 156, rue de Vaugirard, 75737 Paris Cedex 15, France. B. Jabri : Schultz Laboratory, Department of Molecular Biology, Washington road, Princeton University, Princeton, NJ 08540, États-Unis.

E-mail : cerf@necker.fr.

E-mail : bjabri@molbio.princeton.edu

$\mathrm{m} / \mathrm{s} n^{\circ} 11$, vol. 17 , novembre 2001

\title{
La maladie coliaque : une maladie auto-immune induite par un antigène alimentaire
}

La maladie coeliaque est une entéropathie auto-immune induite par un antigène alimentaire, la gliadine, chez des sujets génétiquement prédisposés. Un schéma pathogénique intégrant la liaison génétique aux molécules HLA et l'enzyme cible des auto-anticorps, la transglutaminase, fait jouer un rôle central aux lymphocytes $\mathrm{T} \mathrm{CD4}^{+}$du chorion qui répondent à certains peptides de la gliadine présentés par les molécules HLA-DQ2/8 après désamidation par la transglutaminase. La production des auto-anticorps serait secondaire à la reconnaissance par le système immunitaire de la transglutaminase complexée à la gliadine. Le mécanisme de l'hyperplasie lymphoïde intra-épithéliale, caractéristique de la maladie et à l'origine de complications malignes, n'est pas élucidé. Elle pourrait être déclenchée par un peptide toxique de la gliadine distinct des peptides stimulant les lymphocytes $\mathrm{T} \mathrm{CD}^{+}$, et être secondaire à l'activation de récepteurs de l'immunité innée sur les lymphocytes intra-épithéliaux et à la production de cytokines par les entérocytes modifiés par l'inflammation ou le stress. La poursuite du démembrement des facteurs génétiques et environnementaux s'avère nécessaire pour élucider les mécanismes enclenchant la réaction immune anormale à la gliadine, comprendre la très grande hétérogénéité clinique et histologique de la maladie et peut-être à terme proposer une alternative au régime sans gluten très contraignant.

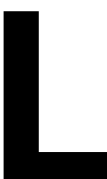

a maladie cœliaque (MC) est une entéropathie induite par les prolamines du blé (gluten) et les protéines apparentées du seigle et de l'orge. La maladie coliaque est une maladie chronique multifactorielle impli- quant des facteurs environnementaux et génétiques. Elle s'apparente moins aux allergies alimentaires qu'aux maladies auto-immunes, auxquelles elle est fréquemment associée. Néanmoins, son expression strictement dépendante d'une expo- 
sition au gluten en fait une exception parmi ces maladies, puisqu'elle est la seule pour laquelle un facteur environnemental clé a été identifié.

La maladie coliaque est observée surtout chez les caucasiens et plus fréquemment chez la femme. Elle se révèle dans la petite enfance par une diarrhée et une cassure de la courbe staturo-pondérale, ou à l'âge adulte par un syndrome de malabsorption souvent plus discret (anémie). De nombreux modes de révélation plus rares ont été décrits: maladies autoimmunes, dermatite herpétiforme, manifestations neurologiques [1]. Le diagnostic est orienté par la présence d'anticorps sériques de type IgG et surtout IgA contre la gliadine et contre un auto-antigène, la transglutaminase tissulaire (tTg) $[1,2]$. Il repose sur la démonstration d'une atrophie villositaire et d'une hyperplasie des lymphocytes intra-épithéliaux (LIE) dans les biopsies intestinales, et sur la régression des anomalies cliniques, biologiques et histologiques sous régime sans gluten [1] (figure 1). L'atteinte intestinale siège au niveau de l'intestin grêle proximal et associe de façon caractéristique une atrophie villositaire subtotale ou totale, une hypertrophie des cryptes, une infiltration lymphocytaire massive de l'épithélium et un infiltrat lymphoplasmocytaire du chorion. Plusieurs études montrent néanmoins une large gamme de lésions intestinales, incluant différents stades d'atrophie villositaire, voire une infiltration lymphocytaire isolée de l'épithélium [1, 3].

La prévalence de la maladie coliaque dans sa forme symptomatique est très variable selon les pays (environ 1/1000-1500 en France), mais les enquêtes sérologiques ont révélé l'existence de nombreuses formes infracliniques dont la fréquence ramènerait la prévalence de la maladie coeliaque à des taux plus comparables dans les différentes pays européens et aux États-Unis (1/200$1 / 400)$. La prévalence augmente dans plusieurs groupes à risque, diabète et maladies auto-immunes, trisomie 21 ou déficit en IgA [1]. Ces données conduisent à représenter les différentes formes de la maladie coliaque à l'intérieur d'un iceberg dont la partie émergée correspond

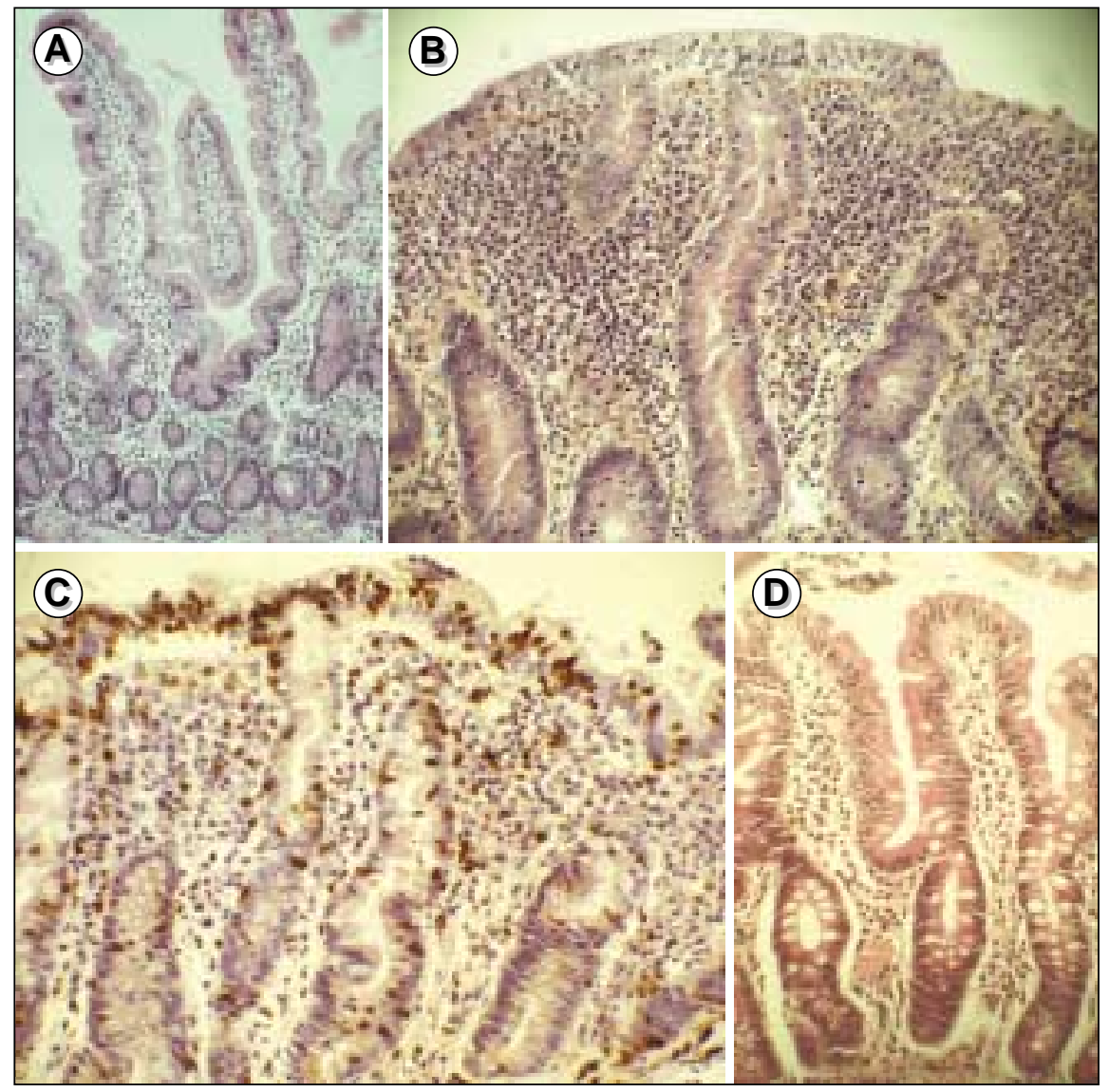

Figure 1. Modifications histologiques au cours de la maladie cœliaque. Biopsies duodénales fixées par le formol et colorées par l'hématéine-éosine $(A, B$, D) ou en immunohistochimie par une technique d'immunoperoxydase (C) (X250). A. Duodénum normal. B. Maladie cœliaque active, typique, caractérisée par une atrophie villositaire totale avec hyperplasie des cryptes, infiltration lymphoplasmocytaire du chorion et infiltration lymphocytaire de l'épithélium. C. Marquage immunohistochimique avec un anticorps anti-CD3 montrant que les lymphocytes $T$ envahissent principalement l'épithélium intestinal au cours de la maladie coliaque active. D. Maladie cœliaque après un an de régime sans gluten: l'efficacité du régime est démontrée par la repousse villositaire et la régression de l'infiltration du chorion. Néanmoins, comme cela est fréquent, il persiste une atrophie villositaire partielle modérée et le nombre des lymphocytes intra-épithéliaux reste augmenté (images dues à la courtoisie du Pr N. Brousse).

Le traitement de la maladie coliaque repose sur un régime d'exclusion très contraignant, mais nécessaire pour prévenir les complications (maladies auto-immunes [4], ostéoporose, infertilité et peut-être complications malignes [1]). Celui-ci reste difficile à faire admettre chez les patients peu ou non symptomatiques, soulignant l'intérêt de mieux comprendre la physiopathogénie des lésions inflammatoires intestinales pour tenter de définir des traitements alternatifs.

\section{Facteurs déclenchants}

Facteurs de prédisposition génétique

La forte prévalence de la maladie coeliaque $(10 \%)$ chez les apparentés au premier degré et la concordance de $70 \%$ entre jumeaux identiques, plus élevée que dans le diabète, soulignent la forte influence de facteurs génétiques. La contribution des gènes HLA est bien démontrée. Plus de $90 \%$ des patients expriment la molécule DQ2 formée par le dimère 


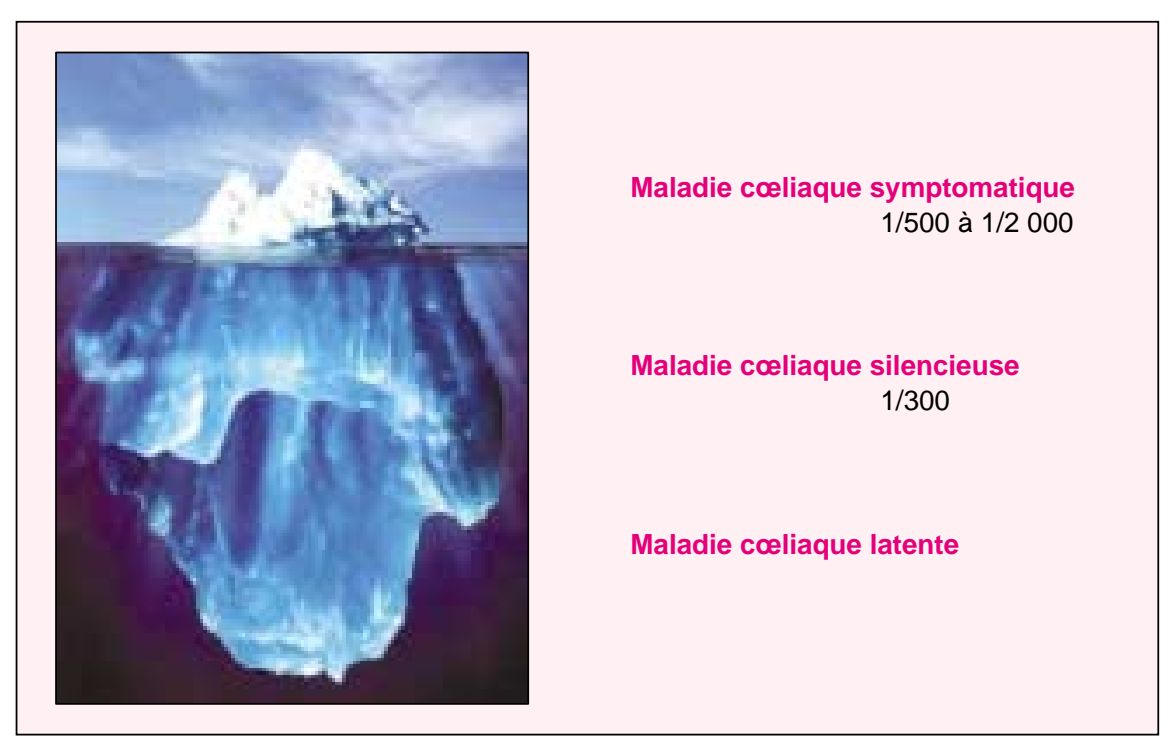

Figure 2. L'iceberg de la maladie coliaque. L'expression clinique très variable de la maladie coliaque a été comparée par A. Ferguson à un iceberg dont la partie émergée correspondrait aux formes symptomatiques [62]. La maladie cœliaque silencieuse se définit par l'absence apparente de manifestations cliniques et la présence d'anticorps anti-gliadine et anti-transglutaminase (appelés anti-endomysium jusqu'à la mise en évidence de l'antigène cible). Elle s'accompagne d'altérations histologiques variables. Sa prévalence a été évaluée par les études sérologiques dans la population générale. La maladie cœliaque latente, mise en évidence grâce aux études familiales, est définie par l'absence de signes cliniques et sérologiques et une réponse histologique anormale (infiltration épithéliale) à une charge en gluten. Les maladies cœeliaques latente et silencieuse peuvent évoluer vers une forme symptomatique.

DQ $\alpha 1 * 0501-Q \beta 1 * 0201$, codé en position cis chez les sujets DR3, ou en trans chez les hétérozygotes DR5/7, un haplotype présent chez $20 \%$ à $30 \%$ des sujets d'origine caucasienne. Un accroissement du risque a été suggéré lorsqu'une seconde copie de l'allèle DQB1*0201 est présente chez les sujets homozygotes DR3-DQ2 ou hétérozygotes DR3-DQ2/DR7-DQ-2. Environ $10 \%$ des patients atteints de maladie coeliaque n'expriment pas DQ2. La plupart de ces patients portent différents sous-types de DR4. Le biais très fort en faveur de l'haplotype DR4-DQ8 conduit la majorité des auteurs à privilégier la molécule $\mathrm{DQ} 8$ (DQ $\alpha 1 * 0301, \beta 1 * 0302)$ comme facteur de susceptibilité chez ces patients [5]. La contribution d'autres gènes situés dans la région HLA est en cours d'étude. Un gène non identifié, en déséquilibre de liaison avec le microsatellite D6S2223 à 2,5 Mb dans la région télomérique au locus HLA-F, modifie de façon comparable la susceptibilité à la maladie cœeliaque et au diabète chez les sujets DR3 [6]. La est associée à l'allèle A, alors que les autres maladies auto-immunes, dont le diabète, sont associés à l'allèle $\mathrm{G}$, le seul à conférer une réponse proliférative $\mathrm{T}$ accrue [10]. La distribution hétérogène des gènes de susceptibilité pourrait contribuer à la variabilité d'expression de la maladie.

\section{Facteurs environnementaux}

Le rôle du gluten dans la pathogénie de la maladie coeliaque a été découvert par Dicke en Hollande lors de ruptures d'approvisionnement en farine de blé au cours de la guerre de 1940-1945. Les études d'épreuve in vitro en culture organotypique et in vivo ont montré que les protéines toxiques sont les prolamines (riches en proline et glutamine), présentes dans le blé, le seigle et l'orge. Les plus étudiées sont les prolamines du blé, classées en plusieurs familles en fonction de leur région amino-terminale, les $\alpha \beta$, $\gamma$ et $\omega$ gliadines et les gluténines. L'activité toxique la mieux établie concerne la large famille des $\alpha \beta$ (ou A-) gliadines, dont la toxicité persiste après digestion par la pepsine et la trypsine (fraction III de Frazer) [11]. La nature des peptides responsables des lésions fait l'objet de nombreux travaux .

Si le gluten est indispensable, d'autres facteurs environnementaux pourraient promouvoir ou au contraire prévenir le déclenchement de la maladie coliaque. Ainsi, une «épidémie» de maladie coeliaque a été observée en Suède chez des enfants de moins de deux ans entre 1985 et 1987 , suivie d'un déclin rapide entre 1995 et 1997 . Ce déclin a coïncidé avec la prolongation de l'allaitement maternel et l'introduction du gluten au cours de l'allaitement [12]. Cependant, on ne sait pas si ces nouvelles pratiques réduisent ou seulement retardent l'apparition de la maladie cœliaque.

Une autre hypothèse ancienne est le rôle déclenchant d'infections intestinales. Le rôle d'une similitude entre peptides de la gliadine et d'un adénovirus n'a finalement pas été retenu. L'hypothèse infectieuse a récemment été renforcée par l'apparition de maladie coeliaque chez des patients traités par l'interféron $\alpha$, une cytokine produite lors d'infections virales, et surtout par la mise en 
évidence de cette cytokine dans l'intestin de patients atteints de maladie coliaque non traités [13]. L'interféron $\alpha$ possède des effets immunomodulateurs qui pourraient favoriser la rupture de la tolérance orale au gluten (voir plus loin).

\section{La maladie cœliaque: conséquence d'une réponse immunitaire intestinale inadaptée au gluten}

La cascade d'événements mise en jeu par le gluten et responsable des lésions intestinales reste discutée. De nombreux arguments plaident en faveur d'un mécanisme immunologique. Ainsi, les lésions épithéliales sont associées à un infiltrat du chorion par des plasmocytes à IgA et des lymphocytes T (LT) CD4 ${ }^{+} \mathrm{CD} 25^{+}$activés [5] et à un infiltrat intra-épithélial massif [14]. Cet infiltrat s'atténue, voire disparaît après exclusion prolongée du gluten, mais peut être réinduit rapidement en réponse à une épreuve in vivo par les peptides toxiques, simultanément à l'augmentation des ARNm codant pour des cytokines pro-inflammatoires [15, 16]. La phase active de la maladie est associée à la production d'anticorps anti-gliadine, et d'auto-anticorps contre une enzyme, la tTg tissulaire [2]. La maladie cœeliaque est fréquemment associée à des maladies auto-immunes [4]. Enfin, la maladie coliaque, comme d'autres maladies inflammatoires ou auto-immunes, est liée aux gènes HLA. Les travaux récents, en établissant des liens entre plusieurs de ces observations, étayent l'hypothèse immunologique.

\section{Rôle des lymphocytes T $C D 4^{+}$du chorion restreints par les molécules HLA DQ2/8 et spécifiques des peptides de la gliadine désamidés par la transglutaminase}

La production de clones $\mathrm{T}$ spécifiques de la gliadine à partir de biopsies intestinales de patients coliaques a permis d'établir des liens entre les molécules HLA-DQ2/8, la tTg, cible des auto-anticorps et la réponse immunitaire $\mathrm{T}$ anti-gliadine (figure

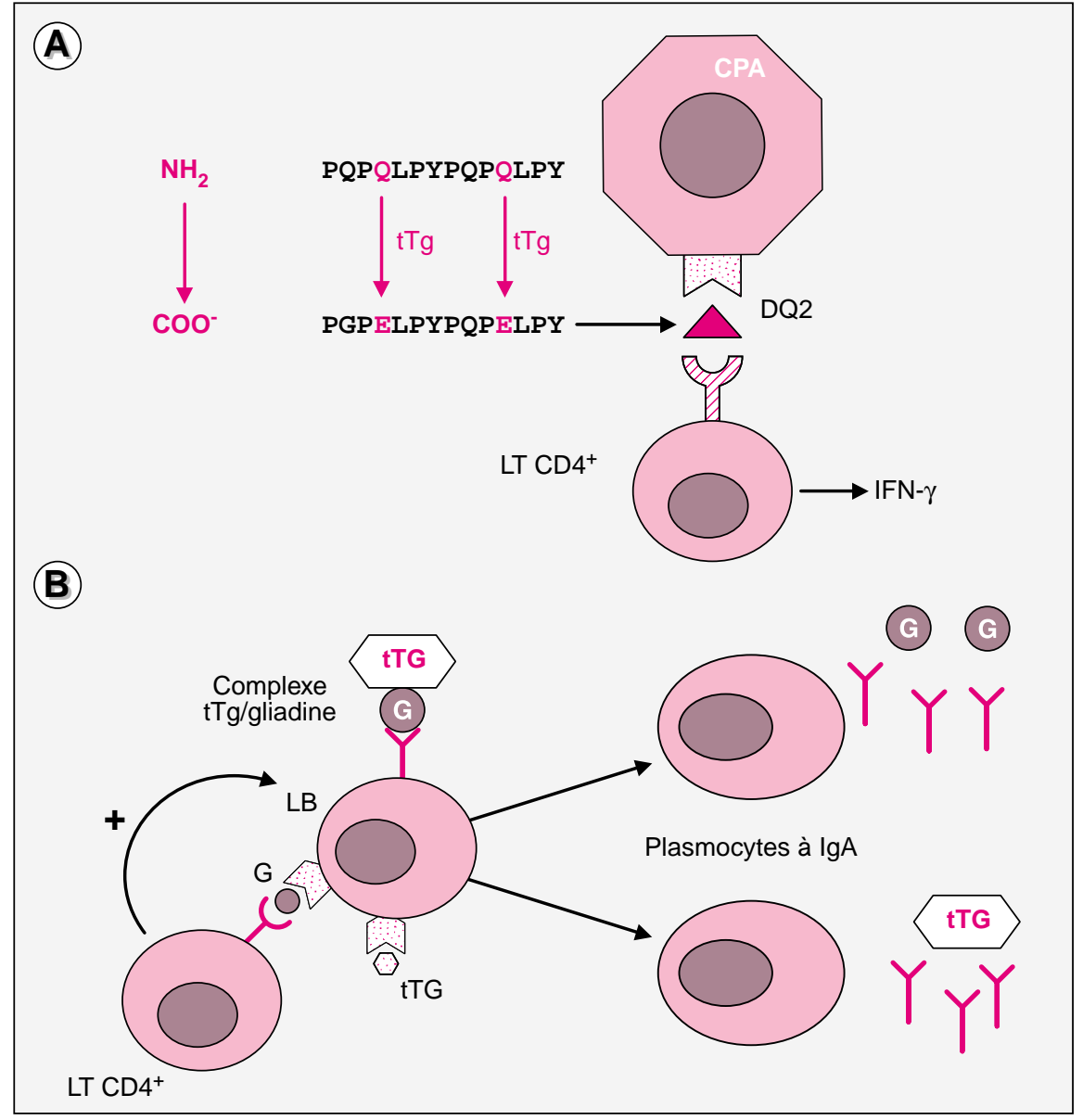

Figure 3. Déclenchement des réponses $T$ anti-gliadine et $B$ anti-gliadine et anti-transglutaminase dans le chorion intestinal. A. Réponse des lymphocytes $T(L T) C D 4^{+}$selon le modèle proposé par L. Sollid [5]. La transglutaminase (tTg), grâce à sa fonction de désamidation, crée des charges négatives au niveau des peptides de la gliadine favorisant leur ancrage dans les poches à peptides des molécules DQ2 et DQ8, qui contiennent des acides aminés chargés positivement. Les molécules HLADQ2/8 et tTg seraient nécessaires pour la présentation des peptides de la gliadine aux $L T C D 4^{+}$par les cellules présentatrices d'antigènes (CPA). Ce modèle ne prend pas en compte l'ensemble des données (voir texte). B. Réponse anticorps. Les anticorps anti-gliadine sont produits grâce aux signaux auxiliaires fournis par les lymphocytes $T \mathrm{CD}^{+}$spécifiques de la gliadine. Les anticorps anti-tTg n'apparaissent qu'en présence de gliadine. La tTg est capable, grâce à sa fonction de catalyse des liaisons entre protéines, de se fixer de façon covalente à la gliadine. La gliadine se comporte alors probablement comme une molécule carrier et permet la production d'anticorps anti-tTg, grâce aux signaux de co-stimulation donnés par les lymphocytes $T$ anti-gliadine aux lymphocytes $B(L B)$ et aux autres CPA présentant à la fois les peptides de la gliadine et de la tTG.

la gliadine obtenus sont en effet $\mathrm{CD} 4^{+}$, et en grande majorité restreints par DQ2 ou DQ8 selon les haplotypes des patients [5]. Chez les sujets DQ2 adultes, la réponse antigliadine pourrait être dirigée principalement contre deux peptides immunodominants partageant les mêmes 7 derniers acides aminés: PQPQLPYPQPQLPY $(\alpha-2$ 62-75) et
QLQPFPQPQLPY ( $\alpha-9$ 57-68) [17, 18]. Cependant, ces peptides n'exercent d'effet stimulant que lorsque la deuxième glutamine du motif QPQ est désamidée en acide glutamique. L'importance de la désamidation dans la reconnaissance des peptides de la gliadine par les lignées ou clones $\mathrm{CD} 4^{+}$dérivés de l'intestin a été attribuée aux caractéristiques de la 
poche à peptides des molécules HLADQ2/DQ8 [5]. Celle-ci permettrait l'ancrage préférentiel de peptides ayant des résidus chargés négativement. Ces résidus sont absents dans les peptides natifs de la gliadine, mais ces derniers sont riches en glutamine désamidable en acide glutamique. La tTg désamide électivement certaines glutamines et, de façon notable, seuls les peptides désamidés dans ces positions peuvent stimuler les clones $\mathrm{T}$ dérivés de l'intestin ou des lymphocytes $\mathrm{T}$ circulant dans le sang de façon transitoire après une stimulation par le gluten $[17,18]$. Ces données plaident en faveur du rôle de LT $\mathrm{CD} 4^{+}$spécifiques du gluten dans le déclenchement de la réponse immune intestinale.

Nilsen et al. ont suggéré que ces lymphocytes pourraient aussi jouer un rôle effecteur central dans la pathogénie de l'atrophie villositaire à travers la sécrétion d'interféron $\gamma$. En effet, la totalité des clones $\mathrm{T} D \mathrm{DQ}$ restreints et spécifiques de la gliadine issus de l'intestin synthétisent de grandes quantités d'interféron $\gamma$, cytokine prédominante dans l'intestin des patients en phase active [19]. Un effet délétère de l'interféron $\gamma$ sur la muqueuse intestinale a été démontré in vitro sur des biopsies humaines [20], et in vivo chez la souris après des injections répétées [21]. Les relais d'action de l'interféron $\gamma$ ne sont pas entièrement élucidés. Il induit l'expression de Fas et du récepteur p55 du TNF (tumor necrosis factor) sur les entérocytes, favorisant ainsi la lyse de ces cellules par des lymphocytes cytotoxiques [21], une voie dont l'importance dans la pathogénie de l'atrophie villositaire est confortée par des études récentes en culture organotypique [22]. L'interféron $\gamma$ active la libération de radicaux libres par les macrophages et les polynucléaires et pourrait aussi favoriser la libération par les macrophages ou le tissu conjonctif de métalloprotéases capables de détruire la trame des villosités. Ce dernier mécanisme d'action reste cependant discuté et semble impliquer plutôt l'IL12 et le TNF [23], deux cytokines dont le niveau d'expression ne serait, curieusement, que peu ou pas augmenté dans les lésions de maladie cœliaque [19].

Cependant, si ce schéma pathogénique est attrayant, il est probable- ment trop simplificateur. Ainsi, une réponse $\mathrm{T}$ anti-gliadine est observée dans le sang périphérique de sujets non HLA-DQ2 /8, et des clones T anti-gliadine restreints par DR ou DP ont été obtenus à partir du sang de sujets atteints de maladie cœliaque ou témoins [24, 25]. En outre, les lymphocytes $\mathrm{T}$ sanguins pourraient répondre préferentiellement contre les peptides non désamidés [26]. Chez la souris, une réponse anti-gliadine est observée dans la plupart des souches, et un peptide immunostimulant induit une réponse comparable chez des souris DQ8 transgéniques, qu'il soit natif ou désamidé. Ainsi, ni les molécules HLA-DQ2/8, ni la désamidation ne semblent nécessaires au déclenchement d'une réponse anti-gliadine. En revanche, la réponse anti-gliadine dans l'intestin semble dirigée préférentiellement contre les peptides désamidés. Ce résultat est probablement à rapporter à la prédominance de ces peptides dans l'intestin. En effet, la désamidation se produit sous l'effet du $\mathrm{pH}$ acide gastrique, et peut-être grâce à la $\mathrm{tTg}$, détectée dans la région sousépithéliale de l'intestin grêle normal et augmentée chez les patients atteints de maladie coliaque en phase active [27, 28]. Molécules HLA-DQ2/8 et désamidation pourraient donc influer sur la nature ou l'intensité de la réponse immune qui intervient dans l'intestin.

Si une réponse anti-gliadine est détectable dans le sang de sujets sains, cette réponse serait observée dans l'intestin exclusivement chez les patients atteints de maladie coeliaque. Ainsi, aucun clone spécifique de la gliadine n'a été obtenu à partir de biopsies intestinales chez 9 sujets témoins [29]. L'intervention d'autres facteurs favorisant une réponse immune intestinale inappropriée à la gliadine est donc vraisemblable (voir plus loin).

Enfin, il faut souligner que les peptides immunodominants chez les patients DQ2 adultes, de même que la totalité des peptides immunostimulant les LT CD4 ${ }^{+}$intestinaux sont curieusement distincts du principal peptide toxique identifié par les études de "challenge" in vivo et in vitro, le peptide $31-59$ de la région amino-terminale des A-gliadines [11, 30]. En outre, les deux peptides immunodominants ne sont pas reconnus par des clones T dérivés de biopsies intestinales d'enfants ayant débuté récemment leur maladie [17]. Ainsi, d'une part, le rôle exact des molécules HLA DQ2/8 et de la tTg dans la constitution du répertoire $T$ anti-gliadine n'est pas entièrement élucidé. D'autre part, l'importance des peptides immunodominants dans la pathogénie de l'atrophie villositaire mériterait d'être vérifiée par des épreuves in vivo en comparant leurs effets à ceux du peptide toxique $31-59$.

\section{Rôle des lymphocytes intra-épithéliaux}

Ferguson et Murray ont été les premiers à décrire l'augmentation massive des lymphocytes associés à l'épithélium intestinal (LIE) au cours de la maladie coeliaque active [14]. La place des LIE dans la pathogénie de la maladie coliaque reste néanmoins controversée, essentiellement parce que les LIE ne semblent pas impliqués dans la reconnaissance directe de la gliadine. L'infiltration intra-épithéliale est donc souvent considérée comme une simple conséquence de l'activation des lymphocytes $\mathrm{T} \mathrm{CD} 4^{+}$ du chorion. Plusieurs arguments plaident contre cette hypothèse. (1) Elle n'est observée ni dans les diarrhées auto-immunes de l'enfant, en dépit d'une infiltration majeure du chorion par des lymphocytes $\mathrm{T}$ activés, ni dans la maladie de Crohn associée à une réaction inflammatoire de type TH1 dans le chorion. (2) Les épreuves in vivo par la gliadine et les études en culture organotypique ont montré que cette infiltration est induite par le peptide toxique 31-59, distinct des peptides reconnus par des lymphocytes $\mathrm{T} \mathrm{CD} 4^{+}$du chorion, mais capable d'induire des lésions épithéliales [30]. (3) L'étude de biopsies organotypiques de patients atteints de maladie coliaque montre que le blocage de la voie CD28 inhibe l'activation des lymphocytes $\mathrm{T}$ du chorion par le gluten et leur synthèse d'interféron $\gamma$, sans prévenir la migration intra-épithéliale des LIE, ni l'expression de Fas par les entérocytes [31]. (4) L'hyperplasie des LIE est le point de départ des proliférations clonales malignes qui compliquent spécifiquement la maladie coeliaque (voir plus loin). 
Rôle de récepteurs de l'immunité innée et de l'interleukine 15 dans l'hyperplasie des LIE

Les études phénotypiques indiquent que les deux sous-populations principales de LIE sont modifiées au cours de la maladie cœliaque: (1) les LIE $\mathrm{CD}^{+}$possédant un récepteur $\mathrm{T} \alpha \beta$ pour l'antigène, augmentés de façon massive exclusivement au cours de la phase active de la maladie; (2) les LIE $\mathrm{T} \gamma \delta$, augmentés de façon variable mais prolongée après exclusion du gluten [32]. L'augmentation des LIE T $\gamma \delta$ ne semble pas permanente, des chiffres normaux ayant été observés, soit dans la période de latence précédant l'apparition d'une maladie coliaque, soit après plusieurs années de régime sans gluten strict [33].

La reconnaissance directe de la gliadine par les LIE est improbable. En effet, le peptide 31-59 peut induire l'infiltration épithéliale, mais aucun clone spécifique de ce peptide ou d'un autre peptide de la gliadine ne semble avoir été obtenu à partir des LIE. En revanche, nos travaux récents suggèrent le rôle de l'interleukine 15 (IL-15) et de récepteurs de l'immunité innée dans les modifications de l'homéostasie des LIE (figure 4). La recherche de nouveaux récepteurs contrôlant les interactions entre LIE et épithélium a en effet montré qu'une fraction importante des LIE humains expriment des récepteurs communs avec les cellules NK (natural killer), que l'expression de deux récepteurs NK est augmentée sur les LIE au cours de la maladie cœliaque et que l'IL-15 contrôle l'expression de ces récepteurs, cette cytokine étant très augmentée au cours de la maladie coliaque [34]. Le premier récepteur NK, CD94, est exprimé sur $30 \%$ des LIE normaux et 70 à $90 \%$ des LIE des patients atteints de maladie coliaque en phase active, ces derniers exprimant la forme activatrice de ce récepteur (faite de CD94 associée à la molécule NKG2C). Le ligand de CD94, la molécule de classe I non classique HLA-E, est induit par l'interféron $\gamma$ sur les entérocytes des patients atteints de maladie coeliaque [34]. L'expression d'un second récepteur NK activateur, NKG2D, est significati-

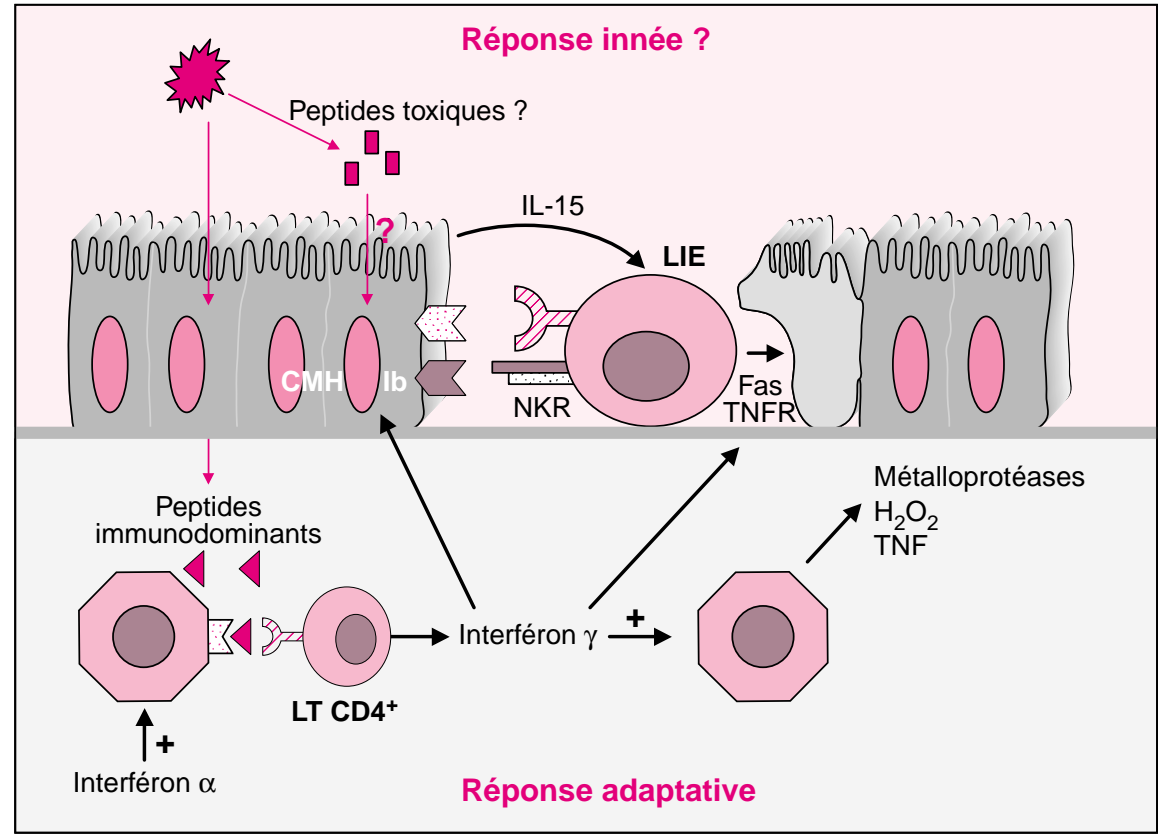

Figure 4. Modèle hypothétique de pathogénie de la maladie cœliaque. Ce modèle tente d'intégrer les données de la littérature et nos données personnelles. La maladie cœliaque est une réponse immunitaire anormale à la gliadine impliquant à la fois le compartiment épithélial et le chorion. Dans l'épithélium, la réponse impliquerait des composants de l'immunité innée avec, $d^{\prime} u n e$ part, dans les entérocytes, hyperproduction d'IL-15 et induction des molécules de classe I non classiques (CMHIb), de Fas et du récepteur du TNF (TNFR), d'autre part, dans les LIE, expression de récepteurs natural killer activateurs (NKR) et du ligand de Fas. Ces modifications pourraient conduire à une activation anormale des LIE et à une destruction des entérocytes en I'absence de reconnaissance directe des peptides de la gliadine par les LIE. Un rôle des peptides dits toxiques dans ces altérations est suggéré par les épreuves in vitro et in vivo. La réponse au niveau du chorion est induite par des peptides distincts qui stimulent directement l'activation des lymphocytes $T C D 4^{+}$. II s'agit d'une réponse adaptative restreinte par les molécules HLADQ2 ou HLA-DQ8. Elle conduit à une hypersécrétion d'IFN $\gamma$, capable d'activer les macrophages et d'induire des altérations épithéliales. Des études récentes suggèrent que l'interféron $\alpha$, sécrété lors d'infections virales, pourrait enclencher cette réponse.

maladie coliaque. NKG2D a pour ligands les protéines de classe Ib MICA/B, induites sur les cellules épithéliales soumises à un stress [35], et de nouveaux ligands, les protéines ULBP dont la localisation et le mode de contrôle ne sont pas encore précisés [36]. Ces deux récepteurs NK pourraient se comporter comme des molécules co-stimulatrices, et abaisser de façon excessive le seuil d'activation du récepteur $\mathrm{T}$ au cours de la maladie coliaque. Il est aussi possible que leur stimulation, seuls ou en synergie, puisse activer directement les LIE.

L'IL-15, outre ces effets sur les récepteurs NK, pourrait contribuer aux modifications de l'homéostasie des LIE en stimulant leur migration [37], leur expansion progressive [38-39], leur production d'interféron $\gamma$ et leur activité cytotoxique [40], ainsi que l'émergence de proliférations malignes [41]. Enfin, l'IL-15 pourrait exercer un effet direct sur les entérocytes et favoriser l'hyperplasie cryptique [42].

Néanmoins, si ces hypothèses fournissent des pistes pour appréhender le mécanisme de l'infiltration intraépithéliale, un travail important reste à effectuer pour cerner les mécanismes à l'origine de la surexpression de l'IL-15, pour délimiter le rôle des récepteurs NK et de leurs ligands, analyser le rôle possible du peptide 31-59 dans les modifications de l'épithélium et établir un lien éventuel avec le terrain génétique. 
Origine de deux complications malignes de la maladie coliaque, sprue réfractaire et lymphome $T$ intestinal à partir des LIE

Des travaux anciens, en cours de réévaluation, indiquent un risque accru de tumeurs malignes au cours de la maladie coeliaque [1]. Outre des adénocarcinomes, les tumeurs les plus caractéristiques sont des lymphomes $\mathrm{T}$ intestinaux, de pronostic sévère. Ces lymphomes expriment CD103, une intégrine spécifique des LIE et présentent un épithéliotropisme, deux caractéristiques qui ont conduit à suggérer leur origine intraépithéliale [43]. Cette hypothèse a été récemment étayée par l'étude de la sprue réfractaire (SR).

La sprue réfractaire est une maladie rare caractérisée par une atrophie villositaire et un syndrome de malabsorption sévère résistant de façon primitive ou secondaire au régime sans gluten. L'étude d'une cohorte de 25 patients a montré que $75 \%$ d'entre eux se rattachent par des critères cliniques, sérologiques et génétiques à la maladie coliaque et se caractérisent par l'expansion massive de LIE anormaux. En effet, ces LIE n'expriment ni récepteur T membranaire, ni le plus souvent de molécule CD8. Ils se rattachent à la lignée $\mathrm{T}$ par l'expression intracellulaire de la chaîne CD3 $\varepsilon$ et des réarrangements de la chaîne $\gamma$ du récepteur $T$. Leur aspect cytologique est normal, mais leur diffusion à l'ensemble du tube digestif et parfois au sang, et le caractère clonal des réarrangements $\mathrm{T} \gamma$ suggèrent qu'il s'agit d'une population maligne ou pré-maligne. Cette hypothèse est renforcée par l'apparition dans $15 \%$ des cas d'un lymphome $\mathrm{T}$ invasif dont la parenté avec la population clonale intra-épithéliale a été démontrée dans deux cas $[44,45]$. Ces données confirment la modification profonde de l'homéostasie des LIE au cours de la maladie cœliaque. Néanmoins, la cascade d'événements impliqués dans leur transformation lymphomateuse progressive reste à élucider. Les travaux en cours suggèrent que les LIE de la sprue réfractaire et les lymphomes T dérivent d'une sous-population minoritaire de LIE normaux. L'émergence d'un clone tumoral pourrait être initialement la conséquence d'événements de recombinaison illégitimes. L'IL-15, produite en grande quantité chez ces patients, et capable d'induire préférentiellement l'expansion de cellules clonales, pourrait alors favoriser l'expansion de ce clone aux dépens des LIE normaux [39].

\section{Rôle des LIE dans la pathogénie de l'atrophie villositaire}

Les LIE synthétisent de l'intérféron $\gamma$ impliqué dans la genèse de lésions épithéliales, et des molécules cytotoxiques (perforine, granzyme B, ligand Fas) dont l'expression est très augmentée dans la maladie coliaque active $[22,46]$. La contribution exacte des LIE à l'entéropathie de la maladie coliaque reste discutée. Néanmoins, une étude chez la souris montre l'induction de lésions épithéliales sévères en réponse à l'activation des LIE [21]. Surtout, l'étude de la sprue réfractaire montre qu'une atrophie villositaire sévère persiste chez des patients qui, du fait du régime sans gluten, n'ont plus a priori de $\mathrm{CD} 4^{+}$activés dans le chorion, mais présentent une infiltration massive de l'épithélium. Il est donc vraisemblable que les LIE activés participent à la destruction des entérocytes et favorisent atrophie villositaire et accélération compensatrice $\mathrm{du}$ renouvellement épithélial (figure 4).

Enfin, LIE $T \gamma \delta$ et $T \alpha \beta$ pourraient exercer des effets différents dans la pathogénie des lésions épithéliales. Certaines études ont suggéré un rôle protecteur des LT $\gamma \delta$ qui pourraient moduler négativement la réponse des T $\alpha \beta$, et réduire les lésions épithéliales en réponse à une infection intestinale [47]. Selon cette hypothèse, il est notable que les LIE T $\gamma \delta$ sont augmentés au cours de la phase de latence et chez quelques patients ayant recouvré une tolérance clinique et histologique, alors que les LIE T $\alpha \beta$ sont exclusivement augmentés pendant la phase active de la maladie coeliaque [32, 48].

\section{Autres mécanismes impliqués dans la pathogénie de la maladie coliaque}

Rôle de la tTg et des auto-anticorps anti-Tg

La tTg est la cible des auto-anticorps caractéristiques de la maladie cœliaque, initialement appelés antiendomysium ou anti-réticuline parce qu'ils révèlent des structures conjonctives sur des coupes de tissu [2]. La tTg possède plusieurs activités enzymatiques. Outre son activité de désamidation, elle peut catalyser la liaison covalente de protéines entre un résidu lysine et un résidu glutamine. Cette activité lui confère un rôle important dans l'apoptose et la formation de la matrice extracellulaire [49]. La tTg peut modifier des protéines endogènes, mais aussi exogènes, et la gliadine est un excellent substrat. L'activité de désamidation de la $\mathrm{tTg}$ favoriseront la reconnaissance de la gliadine par les lymphocytes T (voir plus haut). A l'inverse, la liaison covalente de l'enzyme à la gliadine permettrait sa reconnaissance par le système immunitaire et induirait la production des auto-anticorps, ceux-ci disparaissant sous régime d'exclusion [50] (figure 3B). Plusieurs points restent obscurs: pourquoi la tTg est-elle augmentée chez les patients atteints de maladie cœliaque? S'agit-il d'un phénomène secondaire à l'inflammation, lié à son rôle dans l'apoptose et les remaniements de la matrice extracellulaire [49] ? Les autoanticorps anti-tTg ont-ils un rôle pathogène ? En effet, ceux-ci sont produits dans la muqueuse intestinale [51]. Une étude in vitro suggère qu'ils inhibent la différenciation épithéliale induite par le TGF $\beta$, et attribue cet effet à une diminution de l'activation du TGF $\beta$ par la tTg [52]. Néanmoins, aucune étude n'a pour l'instant démontré un effet des auto-anticorps sur les fonctions enzymatiques de la tTg, et le rôle pathogène des autoanticorps n'est pas établi.

\section{Troubles de la perméabilité intestinale}

Une perméabilité intestinale anormale est observée au cours de la maladie cœliaque [53] et pourrait s'accompagner d'un processing anormal du gluten dans la muqueuse intestinale [54]. L'entrée accrue de gluten, ou de certains de ses peptides, pourrait ainsi favoriser une réponse immune anormale. Néanmoins, les données actuelles suggèrent que ces anomalies sont plus la conséquence des lésions intestinales qu'un facteur de prédisposition. 


\section{Perturbations des mécanismes de tolérance orale}

Dans un modèle murin, l'administration orale de lysozyme à des souris transgéniques, dont tous les lymphocytes $\mathrm{T} \mathrm{CD}^{+}{ }^{+}$sont spécifiques d'un peptide de cette protéine, n'a pas d'effet délétère sur l'intestin. En revanche, une atrophie villositaire est observée lorsque le lysozyme est donné simultanément à un inhibiteur de la production de PGE2, qui perturbe le contrôle immun local en réduisant la production d'IL-10, et en favorisant la prolifération $\mathrm{T}$ et la production de TNF et d'IL-12 [55]. Ces données suggèrent que la présence de lymphocytes $\mathrm{T}$ spécifiques de la gliadine ne suffit pas à induire les lésions intestinales, et que des anomalies du contrôle de la réponse immune intestinale sont aussi nécessaires. Le rôle des lymphocytes $\mathrm{T}$ régulateurs dans le contrôle des réponses immunes intestinales est établi chez la souris. De façon notable, ces lymphocytes partagent des similitudes phénotypiques avec les lymphocytes $\mathrm{T}$ impliqués dans le contrôle de l'auto-immunité $[56,57]$. Une population de lymphocytes $\mathrm{T}$ modulateurs au phénotype similaire $\left(\mathrm{CD} 4^{+} \mathrm{CD} 25^{+} \mathrm{CTLA}^{+}\right)$a récemment été mise en évidence chez l'homme [58]. Il est tentant de suggérer que ces lymphocytes $\mathrm{T}$ jouent un rôle dans le contrôle des réponses immunitaires intestinales chez l'homme et qu'il existe une perturbation de leurs fonctions au cours de la maladie coliaque. Cette hypothèse rendrait compte à la fois de l'inflammation intestinale et de la fréquence des maladies auto-immunes associées. La perturbation des fonctions de ces lymphocytes $\mathrm{T}$ pourrait être déterminée génétiquement ou être provoquée par des facteurs environnementaux, tels que l'interféron $\alpha$ produit au cours d'infections virales [13]. En induisant la maturation des cellules présentatrices d'antigène et la production d'interféron $\gamma$ et d'IL-15, celui-ci pourrait favoriser l'apparition de cellules effectrices Th 1 aux dépens de la population modulatrice $[59,60]$. Le rôle potentiel de l'interféron $\alpha$ dans l'entéropathie de la maladie cœliaque est souligné par une étude très récente en culture

\section{Conclusions}

La maladie coliaque est un modèle privilégié pour étudier les facteurs génétiques et environnementaux impliqués dans le développement des maladies auto-immunes, et les liens entre inflammation et cancer. La production de clones $\mathrm{T} \mathrm{CD}^{+}$restreints par HLA-DQ2/8 et spécifiques de peptides de la gliadine désamidés par la tTg, a permis de proposer un schéma physiopathologique intégrant le principal facteur environnemental, la liaison génétique aux molécules HLA-DQ et l'antigène cible des auto-anticorps. Néanmoins, ces données ne peuvent expliquer l'ensemble de la pathogénie. Plusieurs facteurs de prédisposition génétiques restent à identifier. Les lymphocytes T CD4 intestinaux semblent reconnaître préférentiellement les peptides désamidés par la tTg, mais le rôle précis de la désamidation et de la $\mathrm{tTg}$ reste incertain. La réponse anti-gliadine dans le chorion répond aux critères classiques des réponses immunitaires adaptives, mais l'activation des LIE pourrait impliquer des éléments de la réponse innée, l'IL-15 et les récepteurs NK et molécules de classe I non classiques induites par le stress et l'inflammation. Cependant, les mécanismes induisant cette réponse et ses liens avec la réponse immune spécifique ne sont pas élucidés. La définition des rôles respectifs des peptides stimulant les réponses adaptives $\mathrm{CD} 4^{+}$ dans le chorion, et du peptide toxique 31-59 dont les effets semblent s'exercer plutôt sur le compartiment épithélial, ainsi que la mise en évidence d'autres facteurs favorisant une réponse inadaptée à la gliadine, anomalie de processing ou de contrôle immun, pourraient aider à éclaircir ce point. Un modèle intégrant ces données est proposé dans la figure 4. L'origine intra-épithéliale des proliférations clonales $\mathrm{T}$ qui compliquent la maladie coliaque est maintenant établie. Le rôle favorisant de l'IL-15 est probable, mais la séquence des événements de transformation reste à établir.

La poursuite du démembrement des facteurs génétiques et environnementaux devrait permettre de mieux comprendre les raisons de l'hétérogénéité clinique et histologique de la maladie coliaque. Des études épidémiologiques restent nécessaires pour évaluer l'importance du régime sans gluten dans la prévention des complications et évaluer l'intérêt d'un dépistage systématique des formes silencieuses. Enfin, les travaux physiopathogéniques permettront peutêtre à terme de proposer des traitements alternatifs au régime sans gluten très contraignant. La mise en évidence de peptides immunodominants a suscité l'espoir de développer des blés mutés. Cet espoir doit être tempéré car la délétion d'un peptide immunodominant au sein d'une protéine ne conduit pas nécessairement à éliminer la réponse $\mathrm{T}$ à cette protéine. En outre, le rôle de ces peptides reste à évaluer in vivo

\section{Remerciements}

Nous remercions particulièrement $\mathrm{N}$. Brousse pour ses commentaires et pour sa contribution aux illustrations de ce manuscrit. Nous la remercions ainsi que C. Cellier, J. Schmitz, P. Green, H. Rotterdam, D. Kasarda, les membres de l'équipe EPI-9925 et du laboratoire Schultz pour leur contribution constante aux études menées sur la maladie cœliaque. Nous remercions également les équipes participant au GERMC (Groupe d'Étude et de Recherche sur la maladie coliaque) et au réseau Inserm Progrès.

\section{RÉFÉRENCES}

1. Vahedi K, Bouhnik Y, Matuchansky C. Maladie cœeliaque de l'adulte. Gastroenterol Clin Biol 2001 ; 25 : 485-94.

2. Dieterich W, Ehnis $\mathrm{T}$, Bauer $\mathrm{M}$, et al. Identification of tissue transglutaminase as the autoantigen of celiac disease. Nat Med 1997 ; 3 : 797-801

3. Marsh MN. Gluten, Major histocompatibility complex, and the small intestine. A molecular and immunobiologic approach to the spectrum of gluten sensitivity (celiac sprue). Gastroenterology 1992; 102: 330-54.

4. Ventura A, Magazzu G, Greco L. Duration of exposure to gluten and risk for autoimmune disorders in patients with celiac disease. SIGEP, Study group for autoimmune disorders in celiac disease. Gastroenterology 1999; 117 : 297-303.

5. Sollid LM. Molecular basis of celiac disease. Annu Rev Immunol 2000; 18: 53-81.

6. Lie BA, Sollid LM, Ascher $\mathrm{H}$, et al A gene telomeric of the HLA class I region is involved in predisposition to both type 1 diabetes and coeliac disease. Tissue Antigens $1999 ; 54: 162-8$ 


\section{RÉFÉRENCES}

7. Petronzelli F, Bonamico M, Ferrante $\mathrm{P}$, et al. Genetic contribution of the HLA region to the familial clustering of coeliac disease. Ann Hum Genet 1997; 61 : 307-17.

8. King AL, Yiannakou JY, Brett PM, et al. A genome-wide family-based linkage study of coeliac disease. Ann Hum Genet 2000; 64 479-90.

9. Naluai AT, Nilsson S, Samuelsson L, et al. The CTLA4/CD28 gene region on chromosome 2q33 confers susceptibility to celiac disease in a way possibly distinct from that of type 1 diabetes and other chronic inflammatory disorders. Tissue Antigens 2000; 56 : 350-5.

10. Kouki T, Sawai Y, Gardine CA, Fisfalen ME, Alegre ML, DeGroot LJ. CTLA-4 gene polymorphism at position 49 in exon 1 reduces the inhibitory function of CTLA- 4 and contributes to the pathogenesis of Graves' disease. J Immunol 2000; 165 : 6606 11.

11. Wieser H. Relation between gliadin structure and coeliac toxicity. Acta Paediat 1996; 412 (suppl) : 3-9.

12. Ivarsson A, Persson LA, Nystrom L, et al. Epidemic of coeliac disease in Swedish children. Acta Paediatr 2000: 89: 165-71.

13. Monteleone G, Pender SL, Alstead E Hauer AC, Lionetti P, MacDonald TT. Role of interferon alpha in promoting $\mathrm{T}$ helper cell type 1 responses in the small intestine in coeliac disease. Gut 2001 ; 48: 425-9.

14. Ferguson A, Murray D. Quantitation of intraepithelial lymphocytes in human jejunum. Gut 1971; 12: 988-94

15. Marsh M, Loft D, Garner V, Gordon D. Time/dose responses of coeliac mucosae to graded oral challenges with Frazer's fraction III of gliadin. Eur I Gastroenterol Hepatol $1992 ; 4: 667-73$.

16. Kontakou M, Przemioslo R, Sturgess R, et al. Cytokine mRNA expression in the mucosa of treated coeliac patients after wheat peptide challenge. Gut 1995; 37 : 52 7.

17. Arentz-Hansen $\mathrm{H}$, Korner $\mathrm{R}$, Molberg $\mathrm{O}$, et al. The intestinal $\mathrm{T}$ cell response to alpha-gliadin in adult celiac disease is focused on a single deamidated glutamine targeted by tissue transglutaminase. J Exp Med $2000 ; 191: 603-12$.

18. Anderson RP, Degano P, Godkin AJ, Jewell DP, Hill AV. In vivo antigen challenge in celiac disease identifies a single transglutaminase-modified peptide as the dominant A-gliadin T-cell epitope. Nat Med 2000; 6: 337-42.

19. Nilsen EM, Jahnsen F, Lundin $\mathrm{K}$, et al Gluten induces an intestinal cytokine response strongly dominated by interferon gamma in patients with celiac disease. Gastroenterology 1998; 115 : 551-63.

20. Przemioslo R, Lundin K, Sollid L, Nelufer J, Ciclitira P. Histological changes in small bowel mucosa induced by gliadin sensitive T lymphocytes can be blocked by antiinterferon $\gamma$ antibody. Gut 1995; 36: 874-9.

21. Guy-Grand D, Disanto JP, Henchoz P Malassis-Seris M, Vassalli P. Small bowel enteropathy: role of intraepithelial lymphocytes and of cytokines (IL-12, IFN- $\gamma$, TNF) in the induction of epithelial cell death and renewal. Eur J Immunol 1998; 28: 730-44.

22. Maiuri L, Ciacci C, Raia V, et al. FAS engagement drives apoptosis of enterocytes of coeliac patients. Gut 2001 ; 48: 418-24.

23. Monteleone G, MacDonald T, Wathen N, Pallone F, Pender S. Enhancing lamina propria $\mathrm{TH} 1$ responses with interleukin 12 produces severe tissue injury. Gastroenterology 1999; 117 : 1069-77.

24. Franco A, Appella E, Kagnoff M, et al. Peripheral $\mathrm{T}$ cell response to A-gliadin in celiac disease: differential processing and presentation capacities of Epstein-Barrtransformed B cells and fibroblasts. Clin Immunol Immunopathol 1994; 71 : 75-81.

25. Gjertsen H, Sollid L, Thorsby E, Lundin K. T cells from the peripheral blood of coeliac disease patients recognize gluten antigens when presented by HLA-DR, -DQ, or DP molecules. Scand I Immunol 1994; 39 : 567-74.

26. Molberg O, McAdam S, Korner R, et al. Tissue transglutaminase selectively modifies gliadin peptides that are recognized by gutderived T cells in celiac disease. Nat Med 1998; 4 : 713-7.

27. Bruce S, Bjarnason I, Petratos K. Human jejunal transglutaminase: demonstration of activity, enzyme kinetics, and substrate specificity with special relation to gliadin and coeliac disease. Clin Sci 1985; 68: 573-7.

28. Molberg O, McAdam S, Lundin KE, et al. T cells from celiac disease lesions recognize gliadin epitopes deamidated in situ by endogenous tissue transglutaminase. Eur J Immunol 2001 ; 31 : 1317-23.

29. Molberg O, Kett K, Scott H, Thorsby E, Sollid L, Lundin K. Gliadin specific, HLA DQ2-restricted T cells are commonly found in small intestinal biopsies from coeliac disease patients, but not from controls. Scand J Immunol 1997; 46: 103-9.

30. Sturgess R, Day P, Ellis HJ, et al. Wheat peptide challenge in coeliac disease. Lancet 1994; 343 : 758-61.

31. Maiuri L, Auricchio S, Coletta S, et al. Blockage of T-cell costimulation inhibits Tcell action in celiac disease. Gastroenterology $1998 ; 115: 564-72$.

32. Kutlu T, Brousse N, Rambaud C, Le Deist F, Schmitz J, Cerf-Bensussan N. Numbers of T cell receptor (TCR) $\alpha / \beta^{+}$but not of TCR $\gamma / \delta^{+}$intraepithelial lymphocytes correlate with the grade of villous atrophy in coeliac patients on a long term normal diet. Gut 1993 ; 34 : 208-14.

33. Iltanen S, Holm K, Ashorn M, Ruuska T, Laippala P, Mäki M. Changing jejunal $\gamma \delta \mathrm{T}$ cell receptor (TCR)-bearing intraepithelial lymphocyte density in coeliac disease. Clin Exp Immunol 1999; 117 : 51-5.

34. Jabri B, de Serre NP, Cellier C, et al. Selective expansion of intraepithelial lymphocytes expressing the HLA-E-specific natural killer receptor CD94 in celiac disease. Gastroenterology 2000; 118: 867-79.

35. Groh V, Rhinehart R, Randolph-Habecker J, Topp M, Riddell S, Spies T. Costimulation of CD8alphabeta T cells by NKG2D via engagement by MIC induced on virusinfected cells. Nat Immunol 2001 ; 2 : 255-60.

36. Cosman D, Mullberg J, Sutherland C, et al. ULBPs, novel MHC class I-related molecules, bind to CMV glycoprotein UL16 and stimulate NK cytotoxicity through the NKG2D receptor. Immunity 2001 ; 14: 12333

37. Maiuri L, Ciacci C, Vacca L, et al. IL-15 drives the specific migration of $\mathrm{CD}^{+} 4^{+}$and TCR-gammadelta ${ }^{+}$intraepithelial lymphocytes in organ cultures of treated celiac patients. Am J Gastroenterol 2001 ; 96 : 150-6.

38. Porter B, Malek T. IL-2R $\beta /$ IL-7R $\alpha$ doubly deficient mice recapitulate the thymic and intraepithelial lymphocyte (IEL) developmental defects of $\gamma_{c^{-/}}$mice: roles for both IL-2 and IL-15 in CD8 $\alpha \alpha$ IEL Development. J Immunol 1999; 163: 5906-12.

39. Ku CC, Kappler J, Marrack P. The growth of the very large $\mathrm{CD}^{+} \mathrm{T}$ cell clones in older mice is controlled by cytokines. $J$ Immunol 2001 ; 166: 2186-93.

40. Ebert EC. 1998. Interleukin 15 is a potent stimulant of intraepithelial lymphocytes. Gastroenterology 1998; 115 : 1439-45.

41. Fehniger T, Suzuki K, Ponnapan A, et al. Fatal leukemia in interleukin 15 transgenic mice follows early expansion in natural killer and memory phenotype $\mathrm{CD}^{+} \mathrm{T}$ cells. J Exp Med 2001 ; 193 : 219-31.

42. Maiuri L, Ciacci C, Auricchio S, Brown V, Quaratino S, Londei M. Interleukin 15 mediates epithelial changes in celiac disease. Gastroenterology 2000 ; 119: 9961006.

43. Spencer J, Cerf-Bensussan N, Jarry A, et al. Enteropathy-associated $\mathrm{T}$ cell lymphoma (malignant histiocytosis of the intestine) is recognized by a monoclonal antibody (HML-1) that defines a membrane molecule on human mucosal lymphocytes. Am J Pathol 1988; 132: 1-5.

44. Cellier C, Patey N, Mauvieux L, et al. Abnormal intestinal intraepithelial lymphocytes in refractory sprue. Gastroenterology 1998; 114: 471-9.

45. Cellier C, Delabesse E, Helmer C, et al. Refractory sprue, coeliac disease, and enteropathy-associated T-cell lymphoma. French coeliac disease study group. Lancet 2000; 356: 203-8.

46. Oberhuber G, Vogelsang H, Stolte M, Muthenthaler S, Kummer A, Radaszkiewicz T. Evidence that intestinal intraepithelial lymphocytes are activated cytotoxic $\mathrm{T}$ cells in celiac disease but not in giardiasis. $\mathrm{Am} \mathrm{J}$ Pathol 1996; 148: 1351-7. 


\section{RÉFÉRENCES}

47. Hayday A, Geng L. $\gamma \delta$ cells regulate autoimmunity. Curr Opin Immunol 1997; 9 : $884-9$

48. Iltanen S, Collin P, Korpela M, et al. Celiac disease and markers of celiac disease latency in patients with primary Sjogren's syndrome. Am J Gastroenterol 1999 ; 94 : 1042

49. Piacentini M, Colizzi V. Tissue transglutaminase: apoptosis versus autoimmunity. Immunol Today 1999; 20 : 130-4.

50. Sollid LM, Molberg O, McAdam S, Lundin KE. Autoantibodies in coeliac disease: tissue transglutaminase-guilt by association? Gut 1997; 41 : 851-2.

51. Marzari R, Sblattero D, Florian F, et al. Molecular dissection of the tissue transglutaminase autoantibody response in celiac disease. J Immunol 2001 ; 166: 4170-6.

52. Halttunen T, Maki M. Serum immunoglobulin A from patients with celiac disease inhibits human T84 intestinal crypt epithelial cell differentiation. Gastroenterology 1999 ; 116 : 566-72.

53. Fasano A, Not T, Wang W, et al. Zonulin a newly discovered modulator of intestinal permeability, and its expression in coeliac disease. Lancet 2000 ; 355: 1518-9.

54. Zimmer KP, Poremba C, Weber P, Ciclitira PJ, Harms E. Translocation of gliadin into HLA-DR antigen containing lysosomes in coeliac disease enterocytes. Gut 1995; 36 : 703-9.

55. Newberry RD, Stenson WF, Lorenz RG. Cyclooxygenase-2-dependent arachidonic acid metabolites are essential modulators of the intestinal immune response to dietary antigen. Nat Med 1999; 5 : 900-6.

56. Takahashi T, Tagami T, Yamazaki S, et al. Immunologic self-tolerance maintained by CD25(+) CD $4(+)$ regulatory $\mathrm{T}$ cells constitutively expressing cytotoxic T lymphocyte-associated antigen 4. I Exp Med 2000; 192: 303-10.

57. Read S, Malmstrom V, Powrie F. Cytotoxic $\mathrm{T}$ lymphocyte-associated antigen 4 plays an essential role in the function of CD25(+)CD4(+) regulatory cells that control intestinal inflammation. J Exp Med $2000 ; 192$ : 295-302.

58. Shevach EM. Certified professionals : CD4(+)CD25(+) suppressor T cells. I Exp Med 2001; 193: F41-6.

59. Gallucci S, Matzinger P. Danger signals : SOS to the immune system. Curr Opin Immunol 2001 ; 13 : 114-9.

60. Biron CA. Interferons $\alpha$ and $\beta$ as immune regulators: a new look. Immunity 2001; 14: 661-4.

61. Monteleone G, Pender SLF, Wathen NC, MacDonald TT. Interferon- $\alpha$ drives Tcell mediated immunopathology in the intestine. Eur J Immunol $2001 ; 31: 22-47$.

TIRÉS À PART

N. Cerf-Bensussan.

\section{Summary}

Coeliac disease:

an autoimmune disorder

induced by a dietary antigen

Coeliac disease is an autoimmune enteropathy induced by a dietary antigen, gliadin, in genetically predisposed individuals. The genetic linkage to HLADQ2/8 and the identification of the enzyme transglutaminase as the target for the autoantibodies have led to propound a key pathogenic role for lamina propria HLA-DQ2/8 restricted CD4+ T cells recognizing gliadin peptides deamidated by tranglutaminase. Autoantibody production is ascribed to the recognition of transglutaminase bound to gliadin by the immune system. The mechanism of the intraepithelial lymphoid hyperplasia, which is a hallmark of the disease and gives rise to malignant complications, remains elusive. It may be triggered by a toxic peptide distinct from the peptides stimulating the CD4 lymphocytes, and is likely favoured by the release of cytokines and activation of innate lymphocyte receptors by enterocytes modified by stress and inflammation. Identification of other genetic and environmental factors remains necessary to define the mechanisms triggering the abnormal immune response to gliadin, to understand the rationale for the wide clinical and histological spectrum, and in the long term to design alternative treatments to the current strenuous gluten free diet.

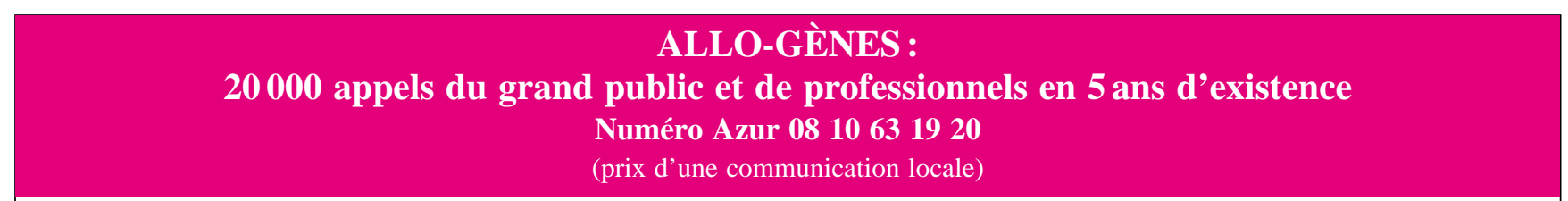

Allo-Gènes, centre national d'information sur les maladies génétiques, est né en 1995 de la réflexion menée par l'Association Française contre les Myopathies (AFM) avec le ministère chargé de la Santé et les généticiens cliniciens.

Il répond aux questions des personnes confrontées à une maladie génétique ou à une maladie rare non génétique et est accessible aux personnes concernées personnellement ou familialement comme aux professionnels de santé (médecins et paramédicaux).

Contact : Professeur Marie-Louise Briard/directrice d'Allo-Gènes - Tél.: 0156538132. 